\title{
Магнитофононные осцилляции магнитосопротивления в квантовой яме InAs/GaSb с инвертированным зонным спектром
}

\author{
() И.В. Кочман, М.П. Михайлова, А.И. Вейнгер, Р.В. Парфенььев \\ Физико-технический институт им. А.Ф. Иофрфе Российской академии наук, \\ 194021 Санкт-Петербург, Россия \\ E-mail: kochman@mail.ioffe.ru
}

Поступила в Редакцию 2 декабря 2020 г.

В окончательной редакции 11 декабря 2020 г.

Принята к публикации 11 декабря 2020 г.

\begin{abstract}
Впервые из спектров микроволнового поглощения в магнитном поле в структуре с квантовой ямой InAs/GaSb с инвертированным зонным спектром выявлены осцилляции магнитосопротивления, обусловленные резонансным рассеянием двумерных электронов на акустических фононах. Индуцированные взаимодействием с акустическими фононами магнитофононные осцилляции магнитосопротивления проявляются в широком интервале температур 2.7-270 К и достигают максимума по амплитуде при $120 \mathrm{~K}$. Рассматриваются электронные переходы между уровнями Ландау с энергией акустических фононов, равной энергии двумерных электронов в InAs с импульсом $2 k_{\mathrm{F}}$. Спектры магнитосопротивления получены на установке электронного парамагнитного резонанса на образцах квантовых ям InAs/GaSb с полуизолирующей подложкой без контактов.
\end{abstract}

Ключевые слова: квантовая яма InAs/GaSb, инвертированный зонный спектр, магнитофононные осцилляции магнитосопротивления, акустические фононы.

DOI: 10.21883/FTP.2021.04.50731.9569

\section{1. Введение}

Гетероструктуры на основе квантовых ям (КЯ) InAs/GaSb представляют интерес для изучения квантоворазмерных эффектов при низких температурах в транспортных явлениях в связи с возможностью создания инвертированной зонной структуры. Бинарные соединения InAs и $\mathrm{GaSb}$ характеризуются сравнительно узкой запрещенной зоной и имеют постоянную решетки, близкую к $6.1 \AA$ [1]. При достижении критической толщины двумерных (2D) слоев (> $85 \AA)$ энергетический спектр KЯ InAs/GaSb меняется от полупроводникового расположения энергетических зон к полуметаллическому (разъединенный гетеропереход II типа), когда уровень $E_{1}$ зоны проводимости InAs располагается ниже уровня валентной зоны $\mathrm{GaSb} H_{1}$ на величину перекрытия, равную $\Delta=150$ мэВ [2]. Это приводит к гибридизации электронно-дырочных состояний в перекрывающихся подзонах размерного квантования [3] с учетом краевых состояний [2], что важно принимать во внимание при исследовании квантового транспорта, включая спиновый эффект Холла [4].

Под воздействием магнитного поля и низких температур в вырожденном двумерном электронном газе наблюдаются осцилляции Шубникова-де Гааза (ШдГ) в магнитосопротивлении при модулировании плотности состояний на уровне Ферми магнитным полем. Также наблюдаются магнитофононные осцилляции [5], обусловленные целым рядом явлений, вызванных резонансным рассеянием электронов как на оптических [6], так и на акустических фононах [7-9].

Магнитофононные осцилляции в полупроводниках изучаются довольно давно $[6,10]$. В последнее время особый интерес представляют осцилляции магнитосопротивления, вызванные резонансным рассеянием электронов между уровнями Ландау при поглощении акустических фононов (phonon-induced resistance oscillations, PIRO), которые наиболее ярко проявляются в 2D структурах с высокой подвижностью носителей заряда в умеренных магнитных полях [7,9].

Роль интерфейсных или объемных акустических фононов в появлении осцилляций магнитосопротивления обсуждалась в обзоре [5].

В данной работе исследуются магнитофононные осцилляции, которые наблюдались в образцах КЯ InAs/GaSb с инвертированным зонным спектром, выращенных на полуизолирующей подложке InAs, без нанесения контактов, в широком температурном интервале $T=2.7-270 \mathrm{~K}$. Температурные зависимости магнитосопротивления структур исследованы на установке электронного парамагнитного резонанса (ЭПР) при разной ориентации внешнего магнитного поля относительно плоскости 2D слоя. В проведенных ранее исследованиях по микроволновому поглощению [11] были показаны возможности разделения осциллирующих вкладов в магнитосопротивление квантованных состояний двумерных и объемных электронов в КЯ $\mathrm{InAs} / \mathrm{GaSb}$ на полупроводящих подложках, для создания которых была использована технология MOVPE (metalorganic vapor phase epitaxy) [12].

\section{2. Образцы и методика измерений}

Квантовая яма InAs/GaSb с инвертированным зонным спектром была выращена методом MOVPE на установке 

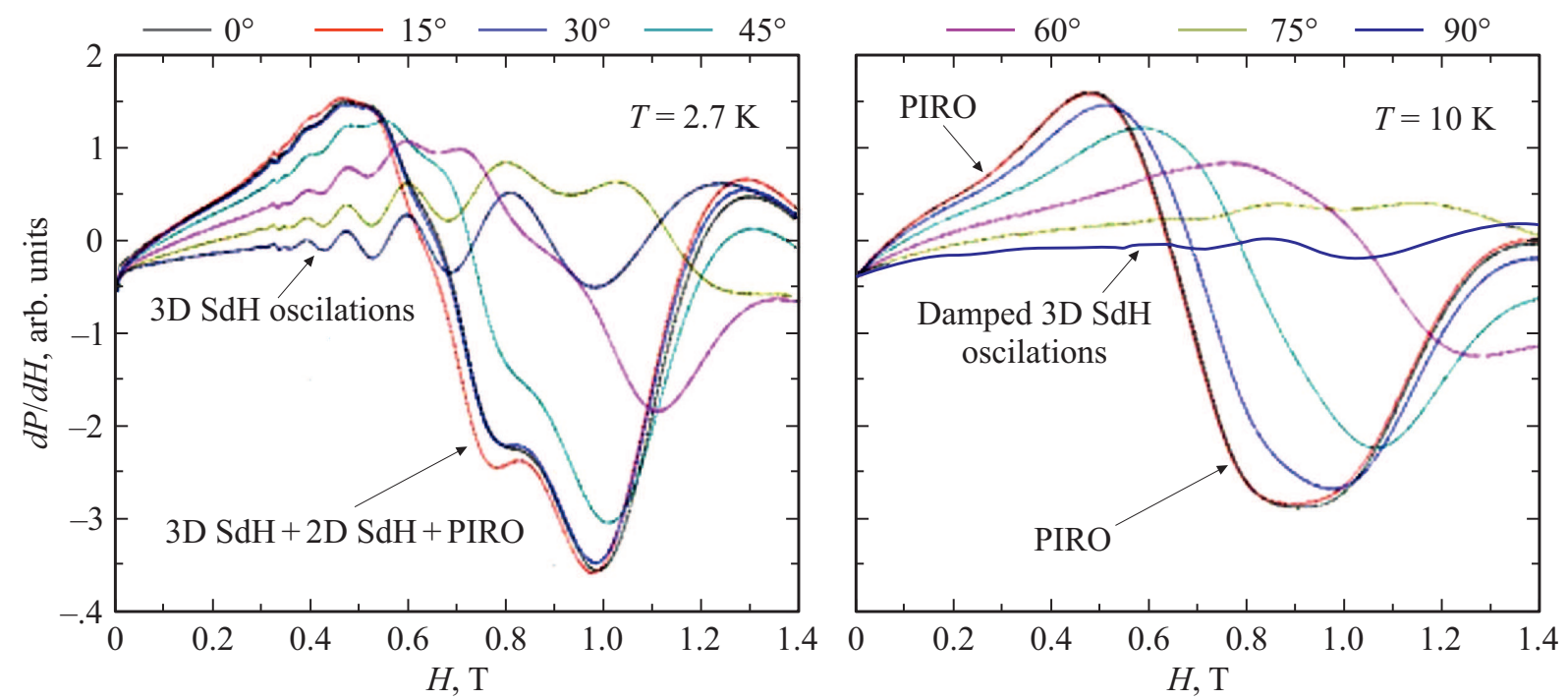

Рис. 1. Угловые зависимости поглощения микроволнового излучения в магнитном поле для температур $2.7(a)$ и $10 \mathrm{~K}(b)$. Сверху вниз по стрелке: $\varphi=0,15,30,45,60,75,90^{\circ}$. Отмечены осцилляции ШдГ в подложке (3D SdH oscillations), комбинированные осцилляции ШдГ от объемных (3D SdH) и двумерных носителей (2D SdH), наложенных на магнитофононные осцилляции PIRO (3D SdH + 2D SdH + PIRO). Небольшие возмущения вблизи 0.35 Тл соответствуют фоновому сигналу от держателя образца.

AIXTRON 200, оборудованной Laytec EPIRAS 200TT RAS (reflectance anisotropy spectroscopy), позволяющей контролировать толщину слоев in situ [12]. Структура двойной квантовой ямы, включающей InAs (12.5 нм) и $\mathrm{GaSb}$ (8нм), ограниченной высокими барьерами AlSb толщиной 30 нм, была выращена на подложке InAs (100) с концентрацией электронов $n=5 \cdot 10^{16} \mathrm{~cm}^{-3}$, с буферным нанослоем InAs (30 нм). Тонкий накрывающий слой $\mathrm{GaSb}$ (6 нм) применялся для защиты слоя $\mathrm{AlSb}$ от окисления. Толщины композитных квантовых ям выбирались из условия получения инвертированной зонной структуры (> $85 \AA$ ) [4], когда первый электронный уровень размерного квантования $E_{1}$ КЯ InAs лежит ниже дырочного уровня $H_{1}$ КЯ GaSb. Размеры образцов составляли $\sim(3 \times 5 \times 10)$ мм.

Исследования были выполнены на установке ЭПР, позволяющей в дополнение к классическому изучению спинового упорядочения и взаимодействия парамагнитных центров в объемных образцах $[13,14]$ проводить исследования изменения магнитосопротивления в двумерных структурах [15]. Эксперимент проводился в широком температурном интервале $T=2.7-270 \mathrm{~K}$ и в квантующих магнитных полях до 1.4 Тл. Угловые зависимости магнитосопротивления были получены вращением образца вокруг оси [100] в магнитном поле.

Образцы КЯ помещались в пучность электромагнитного микроволнового поля объемного резонатора. Развертка магнитного поля (0-1.4 Тл) осуществлялась при постоянной частоте микроволнового излучения $f=9.35$ ГГц. Мощность микроволнового излучения $(P)$, отраженная резонатором, определялась соотношением импедансов волновода и полости резонатора (возможны потери в ее стенках, а также в образце). В непосредственных измерениях эффекта ЭПР максимум потери мощности излучения происходит при перевороте спина, когда за счет энергии электромагнитной волны происходит переход между энергетическими уровнями. Потери мощности при магнитотранспортных измерениях обусловлены тем, что энергия электромагнитной волны используется для ускорения электронов проводимости в магнитном поле.

Спектры поглощения микроволнового излучения (с частотой $f=9.35$ ГГц, энергией кванта $E=\hbar \omega$ $=0.04$ мэВ, где $\omega=2 \pi f$, и мощностью $P=1$ мВт) в постоянном магнитном поле записывались в виде спектров первой производной мощности по магнитному полю $H$, т. е. $d P / d H$, и представлены на рис. 1 .

\section{3. Результаты эксперимента}

На рис. 1 приведены спектры изменения поглощения микроволнового излучения в магнитном поле $(d P / d H)$, полученные на установке ЭПР при $T=2.7 \mathrm{~K}$ для различной ориентации магнитного поля относительно плоскости двумерного слоя. Угол $\varphi=0^{\circ}$ соответствует положению вектора магнитного поля $\mathbf{H} \| z$, где $z-$ направление роста двумерной структуры, а угол $\varphi=90^{\circ}$ соответствует положению внешнего вектора магнитного поля Н $\|$ [010].

Нужно отметить, что при температуре $2.7 \mathrm{~K}$ и угле $\varphi=90^{\circ}$ наблюдались только осцилляции ШдГ от электронов в подложке (3D), которые периодичны в обратном магнитном поле $(1 / H)$ (рис. $2, a)$ и экспоненциально убывают с температурой (рис. 2,b). Из периода осцилляций ШдГ определена концентрация носителей в подложке по формуле

$$
n_{3 \mathrm{D}}=1 / 3 \pi\left(2 m_{e} E_{\mathrm{F}} / \hbar^{2}\right) \approx 10^{16} \mathrm{~cm}^{-3} .
$$



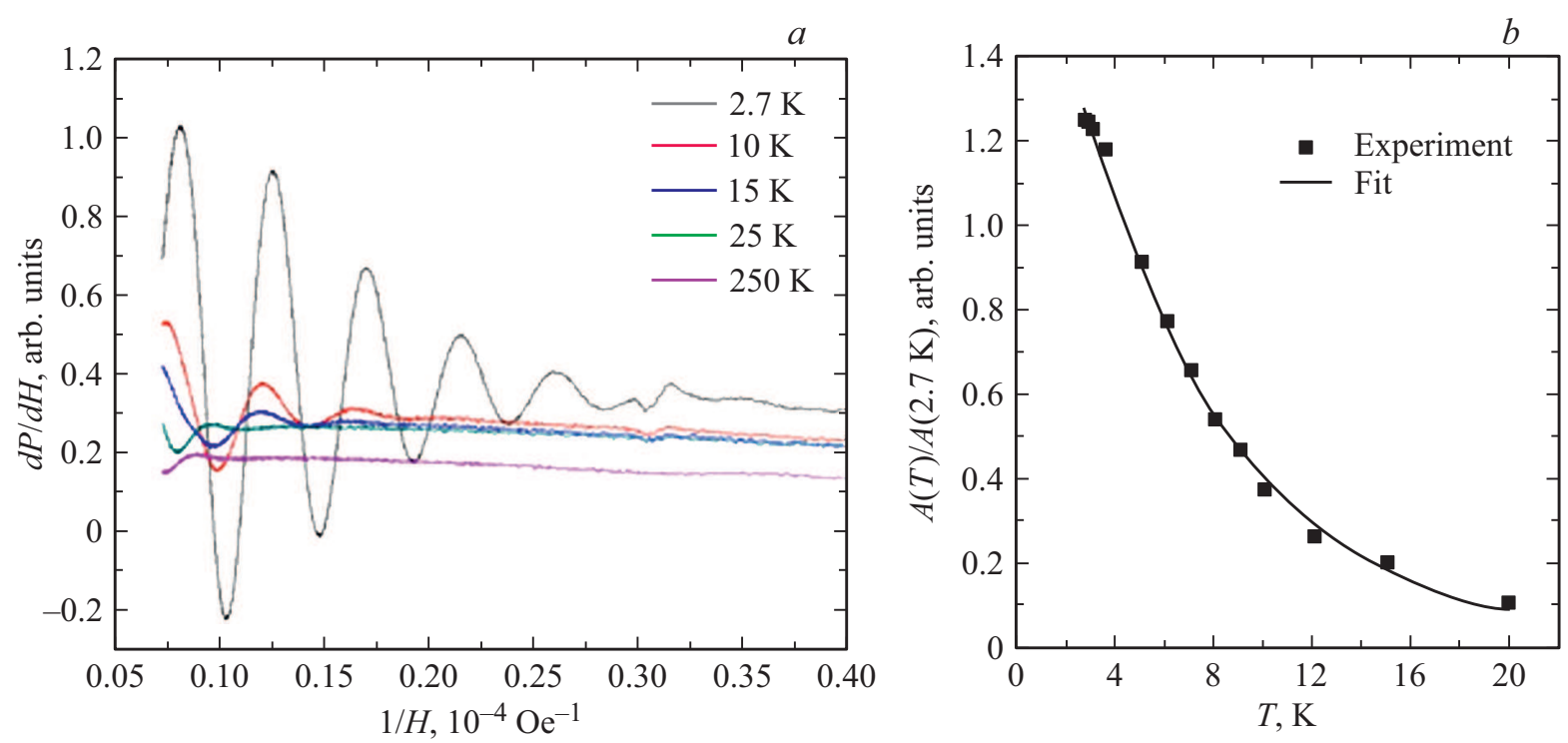

Рис. 2. Осцилляции ШдГ в подложке $(a)$ и их температурная зависимость амплитуды $A(b)$, позволяющая определить эффективную массу. $a$, сверху вниз: $T=2.7,10,15,25,250 \mathrm{~K}$.
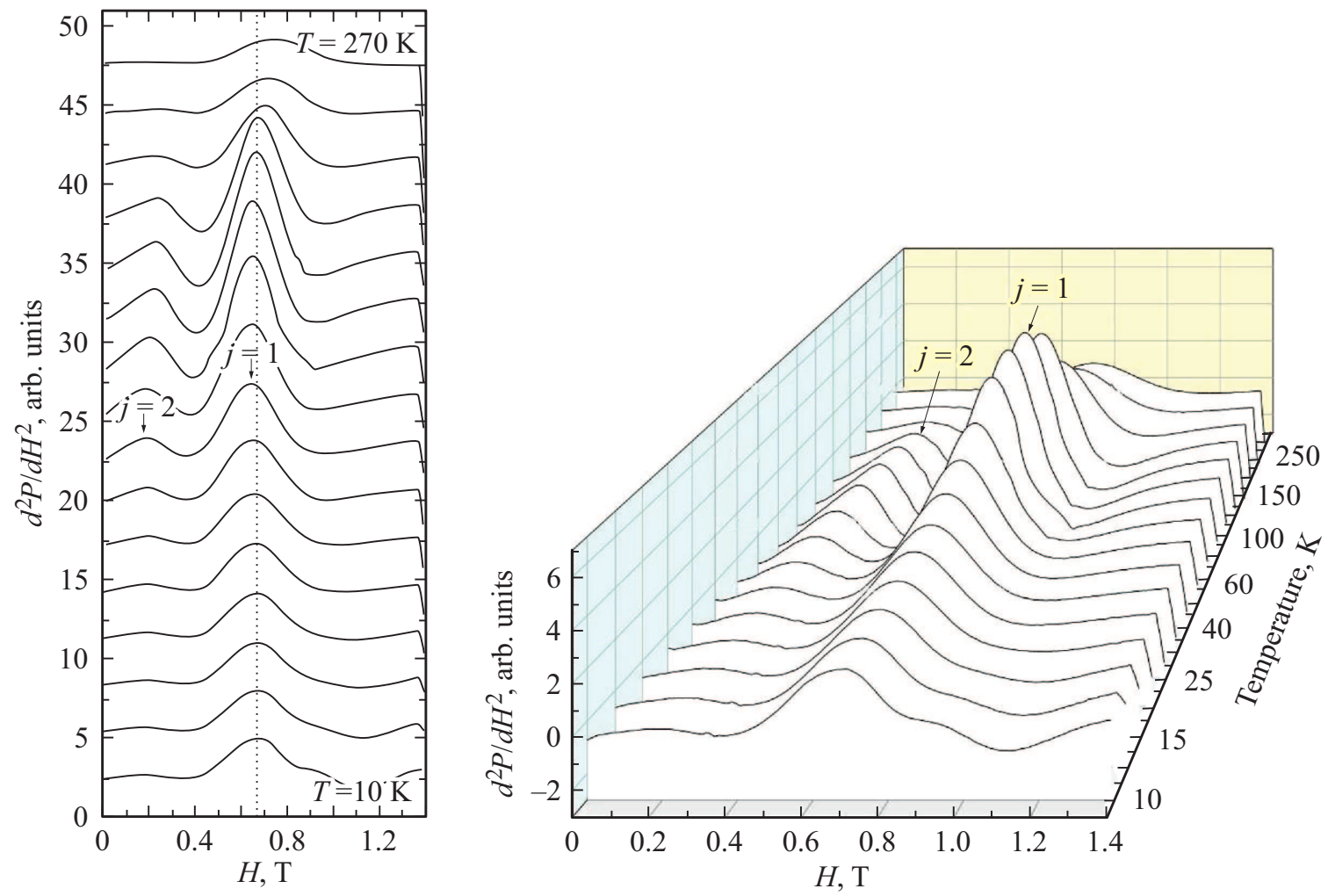

Рис. 3. Температурная зависимость магнитофононных осцилляций второй производной сигнала по магнитному полю $d^{2} P / d H^{2}$. Пунктиром обозначены начальные положения пиков PIRO в магнитном поле. $j$ - максимумы PIRO. Вблизи 0.35 Тл наблюдается фоновый сигнал от держателя для образца.

Эффективная масса электронов на уровне Ферми $E_{\mathrm{F}}$, полученная из анализа температурной зависимости амплитуды осцилляций, равна $m^{*}=0.026 m_{0}$, где $m_{0}-$ масса свободного электрона.

Из рис. 1 следует, что с повышением температуры осцилляции ШдГ от объемных электронов быст- ро убывают по амплитуде. Однако в поле $\sim 0.9$ Тл продолжает проявляться глубокий минимум сигнала, а в поле $\sim 0.3$ Тл наблюдается точка перегиба. Угловая зависимость однозначно указывает на 2D характер обнаруженных особенностей в $d P / d H$ и позволяет связать их с двумерными носителями в КЯ. Учет и разделение вкла- 


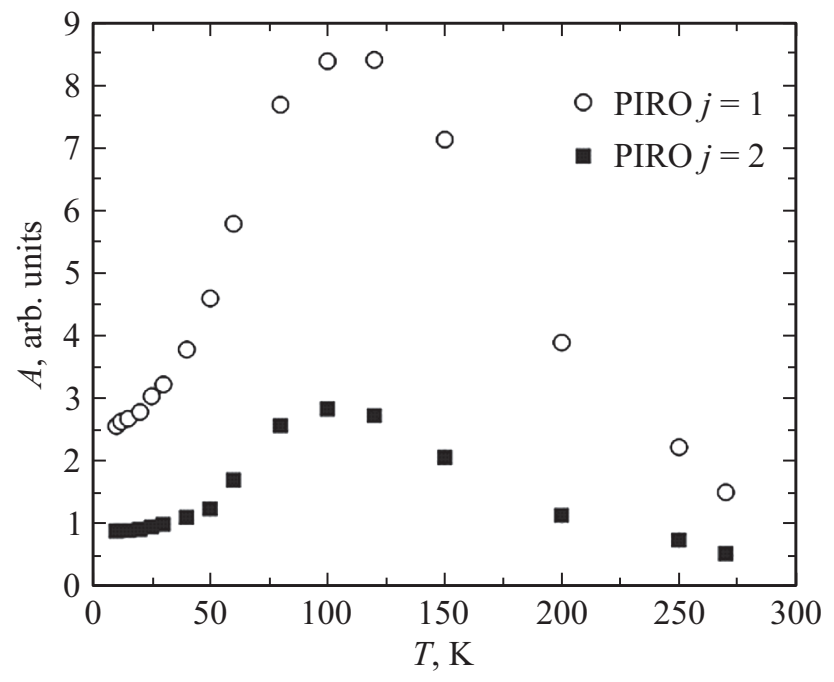

Pис. 4. Температурная зависимость амплитуды магнитофононных осцилляций для двух пиков $(j)$ PIRO.

дов в магнитосопротивление от двумерных и объемных носителей при бесконтактных измерениях $d P / d H$ были представлены нами в работе [11].

Исследования осцилляций ШдГ от двумерных носителей в КЯ при $T=2.7 \mathrm{~K}$, из анализа которых можно было бы получить прямые данные о положении уровня Ферми и эффективной массе носителей, затруднены в силу сильного перекрытия двух наборов частот осцилляций ШдГ - от 2D и 3D носителей. Таким образом, компьютерная обработка данных позволяет лишь качественно подтвердить существование осцилляций ШдГ от двумерных носителей в КЯ, амплитуда которых спадает с температурой быстрее, чем осцилляций от $3 \mathrm{D}$ носителей.

На рис. 3 представлена температурная зависимость второй производной сигнала по магнитному полю, $d^{2} P / d H^{2}$, которая позволяет анализировать относительное изменение сопротивления в магнитном поле с высокой степенью разрешения.

На температурной зависимости магнитосопротивления следует выделить два результата. Во-первых, амплитуда осцилляций растет с температурой, достигая максимального значения вблизи $120 \mathrm{~K}$, а затем убывает близко к зависимости $\propto T^{-3 / 2}$ (рис. 4). В интервале $2.7-120 \mathrm{~K}$ амплитуда сигнала возрастает в 2.5 раза. Во-вторых, положение в магнитном поле двух резонансных пиков магнитосопротивления меняется с температурой, однако пики сдвигаются несимметрично, что свидетельствует об изменении периода осцилляций после прохождения максимума.

\section{4. Обсуждение результатов}

Осцилляции магнитосопротивления, возрастающие с температурой в большом интервале $2.7-270 \mathrm{~K}$, могут быть объяснены резонансным рассеянием двумерных электронов на акустических фононах с волновым вектором $q=2 k_{\mathrm{F}}$, где $k_{\mathrm{F}}-$ волновой вектор электронов вырожденного двумерного газа (PIRO — phonon induced resistance oscillations) $[5,9]$.

Другой класс магнитофононных осцилляций, известных как MIRO - microwave induced resistace oscillations [5], в данной работе не рассматривается, так как энергии микроволнового излучения с частотой $f=9.35$ ГГц и с энергией кванта $E=\hbar \omega=0.04$ мэВ $(\omega=2 \pi f)$ недостаточно для осуществления перехода электрона между уровнями Ландау. Поэтому движение носителей в электромагнитном поле при измерениях магнитосопротивления на установке ЭПР можно рассматривать, как на постоянном токе [13].

Модель взаимодействия вырожденного двумерного электронного газа с фононами в плоскости КЯ, обладающих скоростью $v_{s}=\omega_{s} / q$, была рассмотрена в работах $[8,9]$. Закон сохранения импульса диктует правила отбора, которые позволяют электронам взаимодействовать с акустическими фононами с волновым вектором $q=2 k_{\mathrm{F}}$. При этом резонансное поглощение или излучение фонона с частотой $\omega_{s}=2 k_{\mathrm{F}} v_{s}$ происходит при условии кратности частот $\omega_{s}$ и $\omega_{c}: 2 k_{\mathrm{F}} v_{s}=l \omega_{c}$, $\omega_{c}=e H / m^{*} c$ - циклотронная частота, $l-$ целое число, $l=1,2,3, \ldots, m^{*}$ - эффективная масса электрона. Выражение $2 k_{\mathrm{F}} v_{s}=l \omega_{c}$ определяет положение максимумов магнитофононных осцилляций сопротивления 2D электронного газа.

В работах [7-9] показано, что электрон при движении в квантующем магнитном поле неупруго взаимодействует с фононом с волновым вектором $q=2 k_{\mathrm{F}}$ и совершает непрямые переходы между уровнями Ландау. Точное определение величины волнового вектора Ферми $k_{\mathrm{F}}$ из спектров двумерных осцилляций ШдГ затруднено в силу их сложного перекрытия с трехмерными осцилляциями ШдГ и магнитофононными осцилляциями. Тем не менее, пользуясь выделенными 2D осцилляциями ШдГ, можно дать оценку величины $k_{\mathrm{F}}$, если взять $E_{\mathrm{F}} \approx 10$ мэВ из условия электронейтральности при $n_{2 \mathrm{D}} \approx 10^{11} \mathrm{~cm}^{-2}$, что соответствует экспериментальным данным для нелегированных КЯ InAs/GaSb с инвертированным зонным спектром $[2,16]$ :

$$
k_{\mathrm{F}}=\sqrt{2 m^{*} E_{\mathrm{F}} / \hbar^{2}}
$$

Экспериментальные результаты показывают, что скорость звука в InAs составляет $v_{s}=3-6$ км/с [17]. Если взять стандартные значения эффективной массы для InAs $\left(m^{*}=0.026 m_{0}\right)$, то полученное значение скорости звука будет в несколько раз превышать ранее известные величины.

Выше было отмечено, что особенность структуры КЯ $\mathrm{InAs} / \mathrm{GaSb}$ заключается в инвертированном порядке энергетических зон электронов и дырок на интерфейсе. В точке возможного перекрытия зон должны существовать точки перегиба [18], а на дне зоны проводимости возможно увеличение эффективной массы в результате гибридизации электронно-дырочных состояний [19]. 


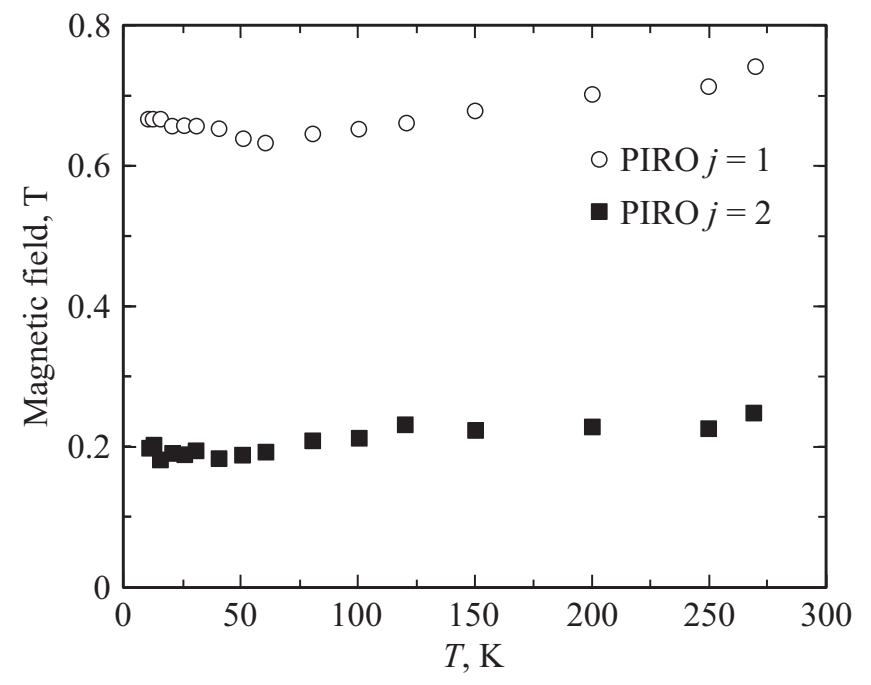

Рис. 5. Положение двух пиков $(j)$ PIRO в магнитном поле и их смещение с температурой.

Таким образом, расхождение в значениях скорости распространения звука и соответственно акустических фононов, рассчитанных из резонансных условий, может быть обусловлено увеличением эффективной массы носителей до значения $m^{*} \approx 0.1 m_{0}$.

Температурная зависимость магнитофононных осцилляций (рис. 4) хорошо согласуется с моделью термически активационного фононного рассеяния - при низкой температуре количество фононов невелико, но их энергии достаточно для того, чтобы электрон переходил на вышестоящий уровень Ландау. С ростом температуры количество термически активированных фононов будет увеличиваться, что приводит к росту амплитуды осцилляций. Как и в случае осцилляций ШдГ, требуется, чтобы выполнялось условие $\mu H>1$, где $\mu-$ подвижность 2D электронов, позволяющее электрону выполнить несколько оборотов по циклотронной орбите в плоскости. Однако при достижении критической температуры размытие уровней Ландау за счет рассеяния и размытия уровня Ферми $2 \mathrm{D}$ электронов (размытие $2 k_{\mathrm{F}}$ ) приводит к падению амплитуды осцилляций с повышением температуры при $T>T_{\max }$. Более того, с повышением температуры из-за различных зависимостей $E_{g}(T)$ в InAs и $\mathrm{GaSb}$ в инверсной области энергетического спектра гетероперехода меняется смещение краев зон, что приводит к эффективному изменению положения уровня Ферми для двумерных носителей и наблюдается на рис. 5 как изменение положения пиков резонанса с температурой. Также с температурой может меняться и заполнение уровней размерного квантования.

\section{5. Заключение}

Нами изучены магнитофононные осцилляции PIRO в 2D структурах InAs/GaSb с высокой подвижностью. Высокое качество структур, выращенных методом MOVPE на полуизолирующей подложке, позволило изучить индуцированные взаимодействием с акустическими фононами магнитофононные осцилляции магнитосопротивления бесконтактным методом микроволнового поглощения в широком температурном интервале $2.7-270 \mathrm{~K}$. Обнаружено, что амплитуда магнитофононных осцилляций магнитосопротивления достигает своего максимального значения при температуре $120 \mathrm{~K}$, а положение пиков зависит от уровня Ферми в области перекрытия зон материалов гетероструктуры с учетом размерного квантования.

\section{Конфликт интересов}

Авторы заявляют, что у них нет конфликта интересов.

\section{Список литературы}

[1] H. Kroemer. Phys. E: Low-Dim. Syst. Nanostr., 20, 196 (2004).

[2] I. Knez, R.-R. Du, G. Sullivan. Phys. Rev. Lett., 107, 136603 (2011).

[3] M.J. Yang, C.H. Yang, B.R. Bennett, B.V. Shanabrook. Phys. Rev. Lett., 78, 4613 (1997).

[4] C. Liu, T.L. Hughes, X.-L. Qi, K. Wang, S.-C. Zhang. Phys. Rev. Lett., 100, 236601 (2008).

[5] I.A. Dmitriev, A.D. Mirlin, D.G. Polyakov, M.A. Zudov. Rev. Mod. Phys., 84, 1709 (2012).

[6] D.C. Tsui, T. Englert, A.Y. Cho, A.C. Gossard. Phys. Rev. Lett., 44, 341 (1980).

[7] P. Kumaravadivel, M.T. Greenaway, D. Perello, A. Berdyugin, J. Birkbeck, J. Wengraf, S. Liu, J.H. Edgar, A.K. Geim, L. Eaves, R. Krishna Kumar. Nature Commun., 10, 3334 (2019).

[8] M.A. Zudov, I.V. Ponomarev, A.L. Efros, R.R. Du, J.A. Simmons, J.L. Reno. Phys. Rev. Lett., 86, 3617 (2001).

[9] A.T. Hatke, M.A. Zudov, L.N. Pfeiffer, K.W. West. Phys. Rev. Lett., 102, 086808 (2009).

[10] A. Firsov, V.L. Gurevich, R.V. Parfeniev, S.S. Shalyt. Phys. Rev. Lett., 12, 660 (1964).

[11] M.P. Mikhailova, A.I. Veinger, I.V. Kochman, P.V. Semenikhin, K.V. Kalinina, R.V. Parfeniev, V.A. Berezovets, M.O. Safonchik, A. Hospodkova, J. Pangrac, M. Zikova, E. Hulicius. J. Nanophot., 10, 046013 (2016).

[12] A. Hospodkova, E. Hulicius, J. Pangrac, F. Dominec, M.P. Mikhailova, A.I. Veinger, I.V. Kochman. J. Cryst. Growth, 464, 206 (2017).

[13] A.I. Veinger, A.G. Zabrodskii, T.V. Tisnek, G. Biskupski. Semiconductors, 32, 497 (1998).

[14] А.И. Вейнгер, А.Г. Забродский, Т.В. Тиснек, С.И. Голощапов. ФТП, 45, 1314 (2011).

[15] H. Linke, P. Omling, P. Ramvall, B.K. Meyer, M. Drechsler, C. Wetzel, R. Rudeloff, F. Scholz. J. Appl. Phys., 73, 7533 (1993).

[16] М.П. Михайлова, В.А. Березовец, Р.В. Парфеньев, Л.В. Данилов, М.О. Сафончик, А. Hospodkova, J. Pangrac, E. Hulicius. ФТП, 51, 1393 (2017).

[17] M. Levinshtein, S. Rumyantsev, M. Shur. Handbook Series on Semiconductor Parameters (World Scientific, 1996). 
[18] Н.С. Аверкиев, В.А. Березовец, М.П. Михайлова, К.Д. Моисеев, В.И. Нижанковский, Р.В. Парфеньев, К.С. Романов. ФТП, 46, 2083 (2004).

[19] K. Nilsson, A. Zakharova, I. Lapushkin, S.T. Yen, K.A. Chao. Phys. Rev. B, 74, 075308 (2006).

Редактор Л.В. Шаронова

\title{
Magnetophonon oscillations of magnetoresistance in broken-gap InAs/GaSb quantum well
}

\author{
I.V. Kochman, M.P. Mikhailova, A.I. Veinger, \\ R.V. Parfeniev \\ loffe Institute, \\ 194021 St. Petersburg, Russia
}

\begin{abstract}
For the first time magnetoresistance oscillations from the spectra of microwave absorption were obtained in a brokengap $\mathrm{InAs} / \mathrm{GaSb}$ quantum well structure with an inverted band alignment. In the wide temperature range $(2.7-270 \mathrm{~K})$ we observed distinct magnetophonon oscillations of magnetoresistance caused by resonant scattering of Landau- quantised Dirac electrons on acoustic phonons in an inverted $\mathrm{InAs} / \mathrm{GaSb}$ quantum well. Here we studied electron transitions between Landau levels with an acoustic phonon energy equal to the two-dimensional electron energy with the momentum $2 k_{\mathrm{F}}$ in InAs. Magnetoresistance spectra were obtained by means of contactless electron spin resonance technique on the quantum well $\mathrm{InAs} / \mathrm{GaSb}$ samples with a semi-insulating substrate.
\end{abstract}

